\title{
Impacto na Rotina Acadêmica pela Utilização de Novas Tecnologias por Estudantes de Medicina do Semiárido Paraibano
}

\author{
Sylvio Elvis da Silva Barbosa ${ }^{1}$; Larissa Araújo do Carmo $^{2}$; Renata Lívia Silva Fonsêca Moreira de Medeiros ${ }^{3}$; \\ Symara Abrantes Albuquerque de Oliveira Cabral ${ }^{4}$; Raimundo Edilberto Moreira Lopes ${ }^{5}$; Maria Nizete Tavares Alves ${ }^{6}$
}

\begin{abstract}
Resumo: O processo educacional vem sofrendo inúmeras mudanças no estilo de ensinar e aprender, ou seja, métodos que facilitem a didática do ensino e, consequentemente, o surgimento de técnicas para assimilar os conteúdos. No entanto, no processo de ensino, os mecanismos de aprendizagem estão se modificando conforme suas necessidades, e é nesse mundo dinâmico, que consideramos como atores principais os alunos e mestres, que ambos são protagonistas na produção de conhecimentos. Atualmente, com o advento das novas ferramentas tecnológicas, como Facebook, WhatsApp e Youtube, na qual são utilizadas como forma de auxiliar no desenvolvimento acadêmico e estreitar, cada vez mais, a relação entre aluno-aluno e aluno-professor. Portanto, espera-se com este conteúdo identificar a importância da utilização desses instrumentos como ferramenta de apoio e desenvolvimento e contribuir como fonte de informação para futuras pesquisas acadêmicas. Objetivo: Verificar se os alunos do curso de medicina da Faculdade Santa Maria - FSM utilizam aplicativos tecnológicas no processo de ensino-aprendizagem. Metodologia: Trata-se de um estudo de campo exploratório, descritivo, com abordagem quantitativa. A pesquisa foi realizada com os acadêmicos do curso de Medicina da FSM, localizada no município de Cajazeiras - Paraíba. A amostra teve como critério a utilização de no mínimo 40\% dos alunos devidamente matriculados na instituição. $\mathrm{O}$ instrumento para coleta de dados compreendeu de um questionário semiestruturado, composto de duas partes, uma relacionada aos dados sociodemográfico e a outra parte questões relacionadas com o objetivo do estudo. A pesquisa obedeceu os princípios éticos, de acordo com a Resolução 466/12 do Conselho Nacional de Saúde. Resultados e Discussões: A amostra contém 145 alunos, inseridos do $2^{\circ}$ ao $9^{\circ}$ período letivo, representando um total de aproximadamente 50,5\% do universo. O gênero feminino, alunos matriculados no $4^{\circ}$ período, faixa etária variando entre $20-25$ anos e com renda familiar entre 1 a 4 salários mínimos, representaram os tópicos com maior prevalência. A maioria dos estudantes, cerca de $84,8 \%$, utilizam algum tipo de ferramenta tecnológica, que possibilitam auxiliar nesse contexto. Além disso, constatou que a maioria desses alunos, cerca de $67,6 \%$, "de vez em quando" utilizam livros da biblioteca, na qual foi comprovado, após realização de um levantamento neste setor, uma diminuição na quantidade de livros retirados mensalmente pelos os mesmos. Já o período de utilização dessas novas ferramentas é entre 2 a 6 horas
\end{abstract}

\footnotetext{
${ }^{1}$ Acadêmico do Curso de Graduação em Medicina pela Faculdade Santa Maria (FSM), Cajazeiras - PB. Possui Graduação em Engenharia Metalúrgica pela Escola de Minas/Universidade Federal de Ouro Preto. Mestre em Engenharia Ambiental - Uso e Conservação de Recursos Hídricos pela Universidade Federal de Ouro Preto;

${ }^{2}$ Acadêmica do Curso de Graduação em Medicina pela Faculdade Santa Maria - FSM, Cajazeiras - PB. Possui Graduação em Enfermagem, pela Universidade Regional do Cariri - URCA;

${ }^{3}$ Doutoranda pela Faculdade de Ciências Médicas Santa Casa- FCMSCSP- Mestre em Enfermagem pela Universidade Federal da Paraíba (2013). Especialista em Saúde Pública pela Faculdade de Ciências Sociais e Aplicadas (2008). Graduada em Enfermagem pela Universidade Federal da Paraíba. Docente da Faculdade Santa Maria;

4 Doutorado em andamento em Ciências da Saúde (Santa Casa da Misericórdia/SP); Mestre em Sistemas Agroindustriais (UFCG); Especialista em Obstetrícia (FSM/CZ); Especialista em Micropolítica da Gestão e Trabalho em Saúde (UFF); Especialista em Gestão da AB e de Redes Microrregionais de Saúde (UFPB); Especialista em Gestão do Trabalho e da Educação na Saúde (UFRN); Especialista em Gestão da Política de Alimentação e Nutrição (FIOCRUZ); Especialista em Saúde da Família (FIP); Graduada em Enfermagem (UFCG); Contato: symara_abrantes@hotmail.com. (83) 99148-6116

5 Mestre e Doutor em Ciências da Educação pela Universidad San Carlos. Especialista em Química e Biologia pela Universidade Regional do Cariri - URCA (2006); Especialista em Gestão Escolar pela Universidade do Estado de Santa Catarina - UDESC (2006); graduado em Pedagogia pela Universidade Estadual Vale do Acaraú - UVA (2002); graduado em Ciências/Química e Biologia pela Universidade Estadual do Ceará - UECE (2002). E-mail: beto.ce@ hotmail.com;

${ }^{6}$ Professora Assistente da Universidade Regional do Cariri - URCA. Curso de Enfermagem. Enfermeira da Secretária de Saúde de Juazeiro do Norte. Doutoranda em Ciências da Saúde na Faculdade de Medicina do ABC.
} 
por dia. Entretanto, os acadêmicos registraram possíveis transtorno associado com o uso demasiado dessas tecnologias, sendo a ansiedade a mais prevalente, cerca de $29 \%$. Conclusão: Observou-se que a maioria dos alunos utilizam algum tipo de mecanismo digital para o seu desenvolvimento intelectual, sendo os mais citados Youtube, WhatsApp e Whitebook. Portanto, é fundamental criar mecanismos oficias entre as instituições de ensino superior, professores e alunos para que esses possam potencializar seu uso de maneira apropriada. Vale citar como exemplo, a utilização desses mecanismos referenciados em faculdades que apresentaram resultados positivos e expressivo diante do processo educacional. Em relação aos distúrbios, foi evidenciado uma taxa de $44,8 \%$ dos entrevistados que percebem algum tipo espécie de dano psíquico.

Palavras-Chave: Educação de Graduação em Medicina. Multimídia. Tecnologia da Informação.

\title{
Impact on the Academic Routine for the use of new Technologies by Students of Semiarid Medicine Paraibano
}

\begin{abstract}
The educational process has undergone numerous changes in the style of teaching and learning, that is, methods that facilitate teaching didactics and, consequently, the emergence of techniques to assimilate contents. However, in the teaching process, the learning mechanisms are changing according to their needs, and it is in this dynamic world that we consider as main actors the students and masters, who both are protagonists in the production of knowledge. Currently, with the advent of new technological tools, such as Facebook, WhatsApp and Youtube, in which they are used as a way to help academic development and increasingly narrow the relationship between student-student and student-teacher. Therefore, it is expected with this content to identify the importance of using these instruments as a tool for support and development and to contribute as a source of information for future academic research. Objective: To verify if the medical students of Santa Maria College - FSM use technological applications in the teaching-learning process. Methodology: This is an exploratory field study, descriptive, with a quantitative approach. The research was carried out with the academics of the Medical School of the FSM, located in the city of Cajazeiras - Paraíba. The sample had as criterion the use of at least $40 \%$ of the students duly enrolled in the institution. The instrument for data collection included a semi-structured questionnaire, composed of two parts, one related to sociodemographic data and the other part related to the study objective. Results and Discussion: The sample contains 145 students, from the 2nd to the 9th academic year, representing a total of approximately $50.5 \%$ of the universe. The female gender, students enrolled in the 4th period, age group ranging between 20-25 years and family income between 1 and 4 minimum wages, represented the topics with the highest prevalence. The majority of the students, about $84.8 \%$, use some kind of technological tool, which they can help in this context. Moreover, he found that the majority of these students, around 67.6\%, "from time to time" use books from the library, which has been proven, after a survey in this sector, a decrease in the amount of books the same. The period of use of these new tools is between 2 to 6 hours per day. However, academics reported potential disorders associated with overuse of these technologies, with anxiety being the most prevalent, about $29 \%$. Conclusion: It was observed that most students use some kind of digital mechanism for their intellectual development, being the most quoted YouTube, WhatsApp and Whitebook. Therefore, it is fundamental to create official mechanisms between higher college, teachers and students so that they can potentiate their use in an appropriate way. As an example, the use of these mechanisms referenced in institutions that presented positive and expressive results before the educational process. Regarding the disorders, a rate of $44.8 \%$ of those interviewed who perceived some type of psychic disorder.
\end{abstract}

Keywords: Education, Medical, Undergraduate. Multimedia. Information Technology.

\section{Introdução}


É notório que o processo educacional vem sofrendo inúmeras mudanças ao longo das décadas. Essas transformações no estilo de ensinar e aprender, ou seja, métodos que facilitem a didática do ensino e, consequentemente, o surgimento de técnicas para assimilar os conteúdos. Contudo, no processo de aprendizagem constante e mútuo, os atores principais são os alunos e mestres, que ambos são protagonistas na produção de conhecimentos. Sendo assim, essa realidade pode ser ratificada segundo o pensamento do autor Paulo Freire (1982) ninguém educa ninguém, ninguém educa a si mesmo, os homens se educam entre si, mediatizados pelo mundo. É nesse contexto que se faz um paralelo entre o pensamento do educador, com o advento da utilização de novas tecnologias interativas, para, então, auxiliar no processo de educação.

De acordo com Teixeira et al. (2015), para Sócrates a função do mestre, é apenas ajudar o discípulo a descobrir, por si mesmo, a verdade. Além disso, ele afirmava que os educadores devem ter paciência com os erros e as dúvidas de seus alunos, pois é a consciência do erro que os leva a progredir na aprendizagem. Desta maneira, o processo de ensino se dava ao ar livre com o mestre no centro e rodeado pelos seus alunos atentos no seu discurso e sempre estimulados a raciocinar.

Com o passar do tempo, a atuação do professor vem sofrendo algumas lapidações, o ambiente de ensino passou a ser limitado por um espaço físico e que utilizava como acessório um quadro negro, assim tornando o aprendizado semelhante a um pedaço de argila úmida que pode ser modelado à vontade do mentor. Além disso, vem ocorrendo uma diminuição da interação entre os alunos, ficando restrito na maior parte do tempo apenas no ambiente acadêmico e/ou a pequenos grupos por afinidades. 
O Ministério da Educação vem estimulando medidas para promover o uso de diversas formas de tecnologia no ambiente escolar, com o intuito de promover a inclusão digital, permitindo auxiliar no desenvolvimento acadêmico e estreitar cada vez mais a relação alunoaluno e aluno-professor. Voltando a forma de interação como antigamente, mas com algumas modificações, ou seja, o ambiente se tornou virtual e o tempo passou a ser ilimitado. Os aplicativos de comunicação, com alcance global, possibilitam realizar de maneira instantânea troca de mensagens pelos celulares, citando como exemplo o WhatsApp, que pode-se criar grupos, enviar imagens, vídeos e áudios. Esse aplicativo alcançou só no ano passado cerca de 800 milhões de novos internautas, com uma média de 1 milhão de novos usuários por dia, chegando a um volume de 30 bilhões de mensagens enviadas diariamente (SMITH, 2016).

Entretanto, Barbosa et al. (2013), relata que a disseminação de novas formas de viver, propiciadas pelo mundo virtual, foi criado um espaço que potencializa manifestações inconscientes, situando-se no intermediário, nem dentro nem fora, nem eu nem você, um espaço "entre". Contudo, o uso das novas tecnologias e as consequências dessa utilização, ainda não são muito claras, dada a obsolescência anunciada dos aparatos, a transitoriedade de seus efeitos e a perda do contato com outras pessoas.

Nesse sentido objetivou-se verificar se os alunos do curso de medicina da Faculdade Santa Maria - FSM utilizam aplicativos tecnológicas no processo de ensino-aprendizagem.

Essa pesquisa possibilitou verificar as vantagens desse tipo de comunicação instantânea, entre os atores principais, e as possíveis desvantagens diante os excessos na manipulação dessas ferramentas.

Cabe salientar também, que essas tecnologias invadiram rapidamente nossas vidas, trazendo necessidade de uso, gerando grandes dependências, para aqueles que almejam estar sempre conectado, e levando ao aprisionamento da atenção pelo usuário, por apresentar diversas oportunidades e entretenimentos.

Portanto, espera-se com este conteúdo identificar a importância da utilização desses instrumentos como ferramenta de apoio e desenvolvimento e contribuir como fonte de informação para futuras pesquisas acadêmicas.

\section{Procedimentos Metodológicos}


Trata-se de um estudo de campo exploratório, descritivo, com abordagem quantitativa. A pesquisa foi realizada com estudantes do curso de Medicina da Faculdade Santa Maria - FSM, localizada no município de Cajazeiras - Paraíba, situado no extremo oeste do estado da Paraíba, distante $477 \mathrm{~km}$ da capital João Pessoa, possuindo uma população aproximada de 58.437 habitantes, ocupando uma área de $586,275 \mathrm{~km}^{2}$, dos quais $28,193 \mathrm{~km}^{2}$ estão em perímetro urbano (IBGE, 2010). Em relação à educação médica, a comarca possui duas instituições de ensino, sendo uma delas a FSM, que foi foco dessa pesquisa, e a outra a Universidade Federal de Campina Grande, campus Cajazeiras.

Os participantes são os próprios estudantes medicina da FSM que utilizam algum tipo de ferramenta tecnológica para o processo de ensino-aprendizagem. Desta maneira, foi escolhida uma amostra aleatória contendo no mínimo $40 \%$ dos alunos devidamente matriculados, de um total de 287, a coleta de dados ocorreu nos meses de outubro a novembro de 2016. Participaram os alunos que atenderam os seguintes critérios de inclusão: aceitar participar da pesquisa, concordar e assinar o Termo de Consentimento Livre e Esclarecido (TCLE), utilizar aplicativos tecnológicos para o seu desenvolvimento de ensino-aprendizagem e possuir celular que possibilita a utilização dessas tecnologias. Critérios de exclusão: não aceitar participar da pesquisa voluntariamente, não utilizar aplicativos tecnológicos para o desenvolvimento de ensino-aprendizagem e não possuir celular que possibilita a utilização dessas novas tecnologias.

Os dados foram analisados no Statistical Package for the Social Sciences (SPSS), versão 21. Além de estatísticas descritivas de frequência (relativa, absoluta, de média, mediana de desvio padrão), adotou-se estatísticas inferências não paramétricas Qui-quadrado de Pearson com correção de Yates, teste de Kruskal-Wallis e teste de correlação de Spearman's rho. Aceitou-se como estatisticamente significativo um $\mathrm{p}<0,05$.

Esta pesquisa está ancorada na Resolução n 466/12 do Conselho Nacional de Saúde (CNS) que visa salvaguardar a autonomia, beneficência, não maleficência, justiça, privacidade e confidencialidade. O estudo "IMPACTO NA ROTINA ACADÊMICA PELA UTILIZAÇÃO DE NOVAS TECNOLOGIAS POR ESTUDANTES DE MEDICINA DO SEMIÁRIDO PARAIBANO" foi submetido ao Comitê de Ética em Pesquisa (CEP) da FSM, que após a aprovação com o parecer de $\mathrm{N}^{\circ}$ 1.603.215 foi realizado a coleta de dados. 


\section{Resultados e Discussões}

Após aplicação dos questionários e com as respostas já tabuladas, foi possível refletir e interpretar os dados visando atingir o objetivo geral da pesquisa. Percebeu-se logo de início que há um uso demasiado dessas novas tecnologias pelos acadêmicos de Medicina.

Diante do exposto, foi iniciado o estudo analisando a descrição dos dados sociodemográfico (sexo, idade, período letivo e renda familiar) dos participantes, na qual observou-se que a maioria da amostra é do sexo feminino $(56,6 \%)$ e com idades entre 20 a 25 anos (56,6\%), ver a Tabela1. A amostra apresentou relativa equidade na distribuição entre os períodos do curso, tendo uma participação maior dos alunos do $4^{\circ}$ período, cerca de $20,7 \%$. Além disso, percebeu-se que quase metade da amostra, aproximadamente 48,3\%, possui renda familiar entre 1 e 4 salários mínimos.

Tabela 1. Descrição dos dados sociodemográfico

\begin{tabular}{|c|c|c|}
\hline & $n$ & $\%$ \\
\hline \multicolumn{3}{|l|}{ Sexo } \\
\hline Masculino & 63 & 43,4 \\
\hline Feminino & 82 & 56,6 \\
\hline \multicolumn{3}{|l|}{ Idade } \\
\hline$<20$ anos & 10 & 6,9 \\
\hline $20-25$ anos & 82 & 56,6 \\
\hline $26-30$ anos & 34 & 23,4 \\
\hline$>30$ anos & 19 & 13,1 \\
\hline \multicolumn{3}{|l|}{ Período } \\
\hline $2^{\circ}$ período & 4 & 2,8 \\
\hline $3^{\circ}$ período & 23 & 15,9 \\
\hline $4^{\circ}$ período & 30 & 20,7 \\
\hline $6^{\circ}$ período & 17 & 11,7 \\
\hline $7^{\circ}$ período & 26 & 17,9 \\
\hline $8^{\circ}$ período & 26 & 17,9 \\
\hline $9^{\circ}$ período & 19 & 13,1 \\
\hline \multicolumn{3}{|l|}{ Renda } \\
\hline 1 a 4 salários mínimos & 70 & 48,3 \\
\hline 5 a 8 salários mínimos & 45 & 31,0 \\
\hline Acima de 8 salários mínimos & 30 & 20,7 \\
\hline
\end{tabular}

Fonte: Autores da pesquisa 
Esse estudo possui uma amostra de 145 alunos, inseridos do $2^{\circ}$ ao $9^{\circ}$ período letivo, representando um total de aproximadamente $50,5 \%$ do universo. Em relação ao perfil sociodemográfico verificou-se que o público feminino, alunos matriculados no $4^{\circ}$ período, faixa etária variando entre 20-25 anos e com renda familiar entre 1 a 4 salários mínimos, representaram os tópicos com maior prevalência. Avila (2014), descreveu em sua pesquisa, que existia uma tendência mundial, até a década de 1960, que a Medicina no Brasil era exercida majoritariamente por homens, mas isso começou a mudar a partir da década de 1970, na qual as faculdades de medicina passaram a ser um espaço de maior circulação das mulheres, aumentando gradativamente ao longo das décadas de 1980 e 1990 e mais aceleradamente na primeira década desse século.

Esse perfil é reforçado no estudo de Scheffer (2013), que evidencia o crescimento da participação feminina no ensino superior, resultando em um maior número de mulheres formadas e inseridas no mercado de trabalho, dados esses obtidos a partir dos Conselhos Regionais de Medicina (CRM).

Em relação a faixa etária dos estudantes pesquisados, demonstrou que esses números convergem com a realidade dos alunos a nível nacional. Essas informações foram confrontadas com os dados obtidos junto a Síntese de Indicadores Sociais (SIS), de 2015, na qual pode-se verificar que em 2014, o percentual maior de estudantes, que frequentavam o ensino superior, foi entre 18 e 24 anos, o que corresponde a um valor de 58,5\%, resultado similar ao descrito nessa pesquisa.

O Instituto Brasileiro de Geografia e Estatística (IBGE) diferencia as classes sociais de acordo com a renda mensal de cada família, nessa ótica os elementos alvo desse estudo foram classificados entre as classes sociais C e D, ou seja, ganham entre 1 a 5 salários mínimos. Além disso, o Instituto mostra que o rendimento nominal mensal domiciliar per capita da população residente no Estado da Paraíba foi de R $\$ 774$ reais em 2015, valores esses que são proporcionalmente similares em comparação a esse estudo.

A Tabela 2 demonstra que apenas 02 (dois) alunos, 1,4\%, não possuem aparelho de celular que possibilita a utilização desses aplicativos. Entretanto, a maioria dos entrevistados, cerca de 98,6\%, possuem esses equipamentos e entre esses, 86,2\%, afirmam que utilizam essas ferramentas no processo de ensino-aprendizagem. 
Tabela 2. Utilização de tecnologias para aprendizagem.

\begin{tabular}{lcc}
\hline & $\boldsymbol{N}$ & $\%$ \\
\hline Possui aparelho celular para novas tecnologias & 143 & 98,6 \\
Sim & 2 & 1,4 \\
Não & 125 & 86,2 \\
Aplicativos tecnológicos no ensino aprendizagem & 20 & 13,8 \\
Sim & & \\
Não & 123 & 84,8 \\
Qual o seu método de estudos & 109 & 75,2 \\
Usa livros & 12 & 8,3 \\
Sites de pesquisa & 14 & 9,7 \\
Artigos científicos & 5 & 3,4 \\
Apostilas do MedCurso e Medcel & 10 & 6,9 \\
Vídeos Aulas & & \\
Transcrições de aulas gravadas
\end{tabular}

Fonte: Autores da pesquisa

Se levar em consideração os dados apresentados por Nagumo (2014), onde cita que a disseminação desses aparelhos celulares é notória, citando como exemplo, o ano de 2013, que foram vendidos mais de 1 bilhão de smartphones no mundo. Um estudo da International Telecommunication Union (2013) estimou que ao final de 2013, haveria 6,8 bilhões de linhas de celulares no mundo, o que quase equivale ao número de pessoas do planeta, o que não significa que todos tenham um aparelho celular devido à desigualdade social e econômica. Há uma estimativa desse estudo de que 2,7 bilhões de pessoas estariam usando a internet no fim do mesmo ano, o que significa que 4,4 bilhões de pessoas ainda não teriam acesso a essa rede. Com a disseminação desses aparelhos na sociedade, é inevitável a ampliação da presença dos celulares na escola. Deste modo, tal fenômeno é visto tanto pela quantidade de aparelhos que foram mensurados na pesquisa quanto pela utilização desses aplicativos pela comunidade acadêmica para o processo de ensino-aprendizagem.

Ao perguntamos sobre os métodos de estudo, os livros são os mais utilizados pelos estudantes, cerca de 84,8\%. Porém, com a popularização dos dispositivos portáteis, principalmente smartphones e tablets, o e-book já é uma realidade no mercado, grandes editoras já possuem versões digitais dos seus livros e revistas mais importantes. Várias escolas e universidades também estão aderindo rapidamente aos benefícios decorrentes do livro digital, tornando-o uma ferramenta essencial para a educação (ALMEIDA; NICOLAU, 
2013). Deste modo, nesse item específico da tabela acima não ficou muito claro se os estudantes utilizam meios digitais para realizar a leitura desses livros.

O intuito de mapear a frequência e preferências de aplicativos, esse estudo indica que mais da metade dos alunos pesquisados, cerca de 67,6\%, refere que "de vez em quando" utilizam livros da biblioteca, mas 68,3\% afirmaram utilizar livros e sites para a realização de trabalhos. Além disso, quando verificam o surgimento de uma nova ferramenta tecnológica de ensino e pesquisa, tendem a esperar o aparecimento de comentários positivos com o propósito de utilizar para tais fins. Percebe-se que os aplicativos mais adotados pelos entrevistados para o desenvolvimento desse processo de ensino-aprendizagem são, respectivamente: Youtube, WhatsApp e Whitebook, ver tabela 3.

Tabela 3. Descrição dos métodos, frequências e preferências de ferramentas de estudo.

\begin{tabular}{|c|c|c|}
\hline & $N$ & $\%$ \\
\hline \multicolumn{3}{|l|}{ Com que frequência utiliza livros da biblioteca } \\
\hline Sempre & 42 & 29,0 \\
\hline De vez em quando & 98 & 67,6 \\
\hline Não utiliza & 5 & 3,4 \\
\hline \multicolumn{3}{|l|}{ Ao realizar um trabalho, sua preferência é } \\
\hline Comprar livros de autores renomados & 4 & 2,8 \\
\hline Pesquisar em sites de consulta & 42 & 29,0 \\
\hline Utiliza livros e sites & 99 & 68,3 \\
\hline \multicolumn{3}{|c|}{ Ao surgir uma nova ferramenta tecnológica, qual a sua reação } \\
\hline Adquirir de imediato & 11 & 7,6 \\
\hline Esperar os comentários positivos & 119 & 82,1 \\
\hline Não tem interesse em possuir & 15 & 10,3 \\
\hline \multicolumn{3}{|c|}{ Quais os aplicativos que você mais utiliza na busca do seu } \\
\hline \multicolumn{3}{|c|}{ desenvolvimento de ensino-aprendizagem } \\
\hline WhatsApp & 91 & 62,8 \\
\hline Whitebook & 33 & 22,8 \\
\hline Youtube & 102 & 70,3 \\
\hline ECG CALC Lite & 1 & 0,7 \\
\hline CID 10 & 1 & 0,7 \\
\hline Essential Anatomy & 1 & 0,7 \\
\hline iSemiologia: Semiologia Médica & 1 & 0,7 \\
\hline Instagram & 2 & 1,4 \\
\hline
\end{tabular}


Pode-se evidenciar nessa pesquisa, que a grande maioria dos estudantes, cerca de $84,8 \%$, utilizam algum tipo ferramenta tecnológica com o propósito de seu desenvolvimento educacional. Nessa perspectiva Kaiesi (2015), aponta os aspectos positivos da utilização, principalmente do WhatsApp na educação, como os apontados por Bansal e Joshi (2014), em que os estudantes consideraram o uso desta ferramenta interessante e pedagogicamente útil. Ngaleka e Uys (2013) indicam uma significativa colaboração e aprendizagem adquirida fora da sala de aula, sem a influência do professor.

Reforçando a tese de que a utilização desse tipo de instrumento, vem ganhando mais espaço diariamente, foi possível verificar no estudo que mais da metade dos alunos pesquisados "de vez em quando" utilizam livros na biblioteca. Corroborando com esses dados, foi realizado um levantamento junto a biblioteca da FSM, com a finalidade de verificar a quantidade de livros retirados mensalmente pelos alunos de Medicina em cada semestre letivo, na qual verificou-se uma diminuição acentuada de locação de livros. Essa observação se iniciou desde o semestre de 2012.2, na qual teve a entrada da primeira turma do referido curso. Comparando cada semestre com a respectiva retirada de livros/por aluno/mês foram apurados os seguintes resultados respectivamente: 2012.2 - 9,9; 2013.1 - 6,5; 2013.2 - 4,9; 2014.1 - 5,1; 2014.2 - 4,3; 2015.1 - 4,3; 2015.2 - 3,7 e 2016.1 - 2, 5 e 2016.2 - 3,3.

Assim, é evidenciado que esse tipo de iniciativa pelos alunos, de buscar novas formas de ferramentas, vem ganhando espaço no seu cotidiano e que impulsionam de maneira indireta as instituições de ensino a se adequarem a esses novos mecanismos. Outro aspecto relevante nesse quesito, com essa diminuição de retirada de livros na biblioteca pelos alunos, o gestor juntamente com o corpo técnico pode dimensionar a quantidade necessária de novas aquisições ou até mesmo inserir outras formas de disponibilizar esse saber.

A tabela 4 exibe que $52,4 \%$ da amostra utilizam essas ferramentas num período entre 2 a 6 horas por dia, ou seja, esse tempo é direcionado exclusivamente para a abordagem acadêmica. Nota-se ainda que a maioria dos alunos pesquisados não percebem que esse período gasto na navegação possa trazer algum tipo de estrago psíquico. Entretanto, para uma parcela dos entrevistados, cerca de $44,8 \%$, é vislumbrada a possibilidade desse uso gerar 
prejuízo a sua vida pessoal, na qual o mais citado foi o de ansiedade $(29,0 \%)$, como evidenciado na Tabela 4.

Tabela 4. Descrição dos períodos e tempos de utilização de ferramentas tecnológicas para a aprendizagem.

\begin{tabular}{lcc}
\hline $\begin{array}{l}\text { Qual o período total diário de utilização dessas novas ferramentas } \\
\text { tecnológicas }\end{array}$ & N \\
De 0 a 2 horas & 39 & 26,9 \\
De 2 a 6 horas & 76 & 52,4 \\
De 6 a 9 horas & 25 & 17,2 \\
Acima de 9 horas & 5 & 3,4 \\
Dentro do contexto da navegação, quanto tempo é direcionado para & & \\
abordagem acadêmica & 46 & 31,7 \\
De 0 a 2 horas & 81 & 55,9 \\
De 2 a 6 horas & 16 & 11,0 \\
De 6 a 9 horas & 2 & 1,4 \\
Acima de 9 horas & & \\
Percebe-se que esse período utilizado pode trazer danos para a vida & & \\
pessoal & 6 & 4,1 \\
Depressão & 42 & 29,0 \\
Transtornos de Ansiedade & 17 & 11,7 \\
TDAH (Transtorno de Déficit de Atenção e Hiperatividade). & 80 & 55,2 \\
Não percebe
\end{tabular}

Fonte: Autores da pesquisa

Entretanto, os dados obtidos em relação aos danos mencionados pelos entrevistados são preocupantes, não só do ponto de vista acadêmico. Teixeira (2016) em seu artigo mencionava que apesar dos inúmeros benefícios da internet, as consequências negativas do seu uso excessivo estão adquirindo destaque em âmbito mundial. Em alguns países como China, Coreia do Sul e Japão, o uso excessivo vem causando dependência, já sendo considerado como uma questão de saúde pública. Segundo uma pesquisa do Instituto Brasileiro de Opinião Pública e Estatística (IBOPE), o Brasil é o terceiro país em ranking mundial de usuários de internet e o primeiro se considerarmos o tempo de acesso à rede (DIAS;RABELO, 2014; AZEVEDO et al., 2014). 
Isso tem estimulado muitos profissionais, como médicos psiquiatras, neurologistas, psicólogos e psicanalistas, a realizarem estudos sobre a dependência da internet. Para especialistas neste assunto, o uso excessivo desse meio de comunicação recebe nomes diferentes: Transtorno de Dependência da Internet (GOLDBERG, 1996); Uso Patológico da Internet (YOUNG, 1998); Uso Problemático da Internet (SHAPIRA et al., 2003).

Nessa pesquisa não tem como distinguir se os participantes já possuíam algum tipo de alteração pré-existente ou se os números mencionados retratam apenas exacerbações dessas manifestações. No estudo realizado por Abreu et al. (2008), já indicava uma possível relação entre a dependência de internet com algumas comorbidades psiquiátricas, sendo listadas a depressão, o transtorno do humor bipolar e os transtornos de ansiedade e TDAH, na qual notou-se que apesar do tempo transcorrido, entre os dois trabalhos, tais alterações psíquicas ainda são citadas.

Desta forma, é importante a realização de ações educacionais no intuito de combater o uso impulsivo e patológico dessas tecnologias. Para reforçar essa constatação Souza (2016), diz que cerca de $95 \%$ dos jovens e adultos brasileiros, com idades entre 15 e 33 anos, se consideram viciados em tecnologia e $63 \%$ utilizam o aplicativo WhatsApp. Existe outra questão a ser considerada: saber se a internet deixou de ser fonte de lazer ou informação e se tornou vício. Para isso, sugere-se observar as mudanças que o uso da internet provocou na vida da pessoa e porque a pessoa fica tantas horas on-line, em busca do quê e com que propósito (BALLONE; MOURA, 2008).

Outro ponto percebido na pesquisa, foi que os alunos não conseguem diferenciar entre o tempo gasto diário com o propósito educacional do período recreativo, visto que eles relatam que navegam a mesma faixa de tempo. Lima (2016), em seu trabalho no ensino fundamental dizia que a distração dos alunos na frente dos computadores, proporcionava a curiosidade dos mesmos em descobrir o novo. Utilizando esse raciocínio, isso pode indicar a utilização simultânea desse mecanismo, pois os alunos podem desviar sua atenção durante os estudos, podemos citar como exemplo o WhatsApp que permite essa possibilidade de fins.

Já a Tabela 5 há uma correlação entre os danos das tecnologias à vida pessoal, com isso verificaram-se associações estatisticamente significativas entre estes dados. Para essa amostra, constatou-se que, proporcionalmente, mais mulheres $(59,8 \%)$, não percebem que as tecnologias trazem danos, assim como a maioria das pessoas não utilizam livros da biblioteca. 
Tabela 5. Associação entre danos das tecnologias à vida pessoal e os dados da pesquisa.

\begin{tabular}{|c|c|c|c|c|c|}
\hline & \multicolumn{5}{|c|}{$\begin{array}{l}\text { Percebe-se que esse período utilizado pode trazer } \\
\text { danos para a vida pessoal }\end{array}$} \\
\hline & \multicolumn{3}{|c|}{ Transtornos de } & \multirow{2}{*}{$\begin{array}{c}\text { Não } \\
\text { percebe }\end{array}$} & \multirow{2}{*}{$p^{*}$} \\
\hline & Depressão & Ansiedade & TDAH & & \\
\hline \multicolumn{6}{|l|}{ Sexo } \\
\hline Masculino & $3(4,8 \%)$ & $20(31,7 \%)$ & $9(14,3 \%)$ & $31(49,2 \%)$ & \multirow{2}{*}{0,63} \\
\hline Feminino & $3(3,7 \%)$ & $22(26,8 \%)$ & $8(9,8 \%)$ & $49(59,8 \%)$ & \\
\hline \multicolumn{6}{|l|}{ Aplicativos tecnológicos } \\
\hline \multicolumn{6}{|l|}{ no ensino aprendizagem } \\
\hline Sim & $5(4,0 \%)$ & $36(28,8 \%)$ & $15(12,0 \%)$ & $69(55,2 \%)$ & \multirow{2}{*}{0,99} \\
\hline Não & $1(5,0 \%)$ & $6(30,0 \%)$ & $2(10,0 \%)$ & $11(55,0 \%)$ & \\
\hline \multicolumn{6}{|l|}{ Com que frequência } \\
\hline \multicolumn{6}{|l|}{ utiliza livros da biblioteca } \\
\hline Sempre & $3(7,1 \%)$ & $11(26,2 \%)$ & $6(14,3 \%)$ & $22(52,4 \%)$ & \multirow{3}{*}{0,60} \\
\hline De vez em quando & $3(3,1 \%)$ & $31(31,6 \%)$ & $10(10,2 \%)$ & $54(55,1 \%)$ & \\
\hline Não utiliza & $0(0,0 \%)$ & $0(0,0 \%)$ & $1(20,0 \%)$ & $4(80,0 \%)$ & \\
\hline \multicolumn{6}{|l|}{$\begin{array}{l}\text { Ao realizar um trabalho, } \\
\text { sua preferência é }\end{array}$} \\
\hline $\begin{array}{l}\text { Comprar livros de autores } \\
\text { renomados }\end{array}$ & $0(, 0 \%)$ & $1(25,0 \%)$ & $1(25,0 \%)$ & $2(50,0 \%)$ & \multirow{3}{*}{0,21} \\
\hline $\begin{array}{l}\text { Pesquisar em sites de } \\
\text { consulta }\end{array}$ & $2(4,8 \%)$ & $18(42,9 \%)$ & $6(14,3 \%)$ & $16(38,1 \%)$ & \\
\hline Utiliza livros e sites & $4(4,0 \%)$ & $23(23,2 \%)$ & $10(10,1 \%)$ & $62(62,6 \%)$ & \\
\hline \multirow{2}{*}{\multicolumn{6}{|c|}{$\begin{array}{l}\text { Ao surgir uma nova } \\
\text { ferramenta tecnológica, } \\
\text { qual a sua reação }\end{array}$}} \\
\hline & & & & & \\
\hline Adquirir de imediato & $1(9,1 \%)$ & $3(27,3 \%)$ & $0(0,0 \%)$ & $7(63,6 \%)$ & \multirow{3}{*}{0,80} \\
\hline $\begin{array}{l}\text { Esperar os comentários } \\
\text { positivos }\end{array}$ & $5(4,2 \%)$ & $34(28,6 \%)$ & $15(12,6 \%)$ & $65(54,6 \%)$ & \\
\hline $\begin{array}{l}\text { Não tem interesse em } \\
\text { possuir }\end{array}$ & $0(, 0 \%)$ & $5(33,3 \%)$ & $2(13,3 \%)$ & $8(53,3 \%)$ & \\
\hline
\end{tabular}

*Teste Qui-quadrado de Pearson com correção de Yates

Fonte: Autores da pesquisa

Analisando essas informações listadas acima, podemos citar o trabalho de Oliveira (2014), onde menciona sobre a própria percepção dos dependentes de internet que o vício afetava a qualidade de vida, no diz respeito aos domínios físico, psicológico, nível de 
independência, relações sociais, meio ambiente e espiritualidade. Verificou-se também que as mulheres utilizam a internet para se acalmar mais do que os homens. Logo, esse estudo reforça que pela própria percepção do usuário e aliado com a finalidade de uso, principalmente, pelas mulheres, em comparação com o gênero masculino, pode resultar uma menor taxa de desenvolver qualquer tipo de transtorno psíquico.

A Tabela 6 é vista uma relação entre os entrevistados que associaram um quadro de depressão, por uso desses aplicativos, com um valor maior na mediana de idade, cujo valor obtido é de 2,50. Outro ponto observado na mesma tabela, é que os alunos que não percebem correlação entre o uso dos aplicativos com a possibilidade de desencadear algum tipo de alteração de ordem psíquica apresentam uma maior mediana de período, ou seja, são os estudantes que estão mais adiantados no curso. Porém, estes resultados não apresentaram diferenças estatisticamente significativas.

Tabela 6. Comparação entre as diferentes percepções de danos que as tecnologias podem causar entre os dados do estudo.

\begin{tabular}{|c|c|c|c|c|c|c|}
\hline \multicolumn{2}{|c|}{$\begin{array}{l}\text { Percebe-se que esse período } \\
\text { utilizado pode trazer danos para a } \\
\text { vida pessoal }\end{array}$} & Idade & Período & Renda & $\begin{array}{l}\text { Período } \\
\text { diário de } \\
\text { utilização }\end{array}$ & $\begin{array}{c}\text { Tempo } \\
\text { direcionado a } \\
\text { abordagem } \\
\text { acadêmica }\end{array}$ \\
\hline \multirow{4}{*}{ Depressão } & Média & 2,50 & 3,00 & 2,17 & 2,17 & 2,17 \\
\hline & $\begin{array}{l}\text { Desvio } \\
\text { padrão }\end{array}$ & 1,04 & 1,09 & 0,75 & 0,75 & 0,75 \\
\hline & Mediana & 2,50 & 3,00 & 2,00 & 2,00 & 2,00 \\
\hline & Média & 2,40 & 5,07 & 1,76 & 2,02 & 1,83 \\
\hline \multirow[t]{2}{*}{$\begin{array}{l}\text { Transtornos de } \\
\text { Ansiedade }\end{array}$} & $\begin{array}{l}\text { Desvio } \\
\text { padrão }\end{array}$ & 0,79 & 2,04 & 0,75 & 0,60 & 0,66 \\
\hline & Mediana & 2,00 & 5,00 & 2,00 & 2,00 & 2,00 \\
\hline \multirow{4}{*}{$\begin{array}{l}\text { TDAH (Transtorno } \\
\text { de Déficit de } \\
\text { Atenção e } \\
\text { Hiperatividade). }\end{array}$} & Média & 2,59 & 4,59 & 1,53 & 1,94 & 2,00 \\
\hline & $\begin{array}{l}\text { Desvio } \\
\text { padrão }\end{array}$ & 0,93 & 2,09 &, 71 & 1,14 & 0,61 \\
\hline & Mediana & 2,00 & 5,00 & 1,00 & 2,00 & 2,00 \\
\hline & Média & 2,40 & 5,07 & 1,71 & 1,94 & 1,75 \\
\hline \multirow[t]{3}{*}{ Não percebe } & $\begin{array}{l}\text { Desvio } \\
\text { padrão }\end{array}$ & 0,77 & 2,29 & 0,81 & 0,75 & 0,68 \\
\hline & Mediana & 2,00 & 6,00 & 1,00 & 2,00 & 2,00 \\
\hline & $p$-valor* & 0,84 & 0,13 & 0,39 & 0,86 & 0,30 \\
\hline
\end{tabular}

*Teste de Kruskal-Wallis 
Fonte: Autores da pesquisa

Os dados obtidos na tabela acima não apresentaram diferenças estatisticamente significativas entre as diferentes percepções de danos que as tecnologias podem acarretar. Mas, alguns estudos sugerem que os usuários podem compensar a baixa auto-estima, a falta de habilidade social, a solidão e a depressão por meio da internet. Isto torna seu uso extremamente reforçador e aumenta a vulnerabilidade para essas pessoas desenvolveram um transtorno relacionado ao uso excessivo da internet (PIROCA, 2012).

Mesmo que os resultados obtidos não possam confirmar alguma relação entre idade e vícios. Entretanto, eles nos indicam uma possível analogia entre os alunos com idade mais avançada ser menos susceptíveis ao desejo incontrolável de permanecer conectado. Reforçando essa tese, outros trabalhos apontam que os jovens mostraram muito mais sintomas de vício do que pessoas mais velhas (SETZER, 2014).

\section{Conclusão}

Respondendo ao objetivo proposto para este estudo de identificar o impacto na rotina acadêmica pela utilização de novas tecnologias por estudantes de medicina do semiárido paraibano, chegou-se à conclusão de que é evidente que a maioria dos alunos de Medicina da FSM utiliza algum tipo de aplicativo digital para o seu desenvolvimento intelectual, isso é demonstrado através dos resultados obtidos das respostas dos questionamentos apurados e pela diminuição a cada semestre de retirada de livros pelos alunos.

Em relação aos aplicativos mais mencionados que os alunos utilizam para o processo de desenvolvimento educacional foram respectivamente: Youtube, WhatsApp e Whitebook. Vale ressaltar que para 55,9\% dos entrevistados, os alunos utilizam esses meios digitais entre 2 a 6 horas por dia com a finalidade de ensino-aprendizagem. Já para o uso recreativo, cerca de 52,4\% dos entrevistados relatam que navegam pelo mesmo período. Essa similaridade nos tempos empregados, pode indicar sobreposição de intenção, pois tanto o Youtube quanto o WhatsApp possibilitam esse fim. 
Deste modo, é fundamental criar mecanismos oficias entre IES, professores e alunos para que possam potencializar seu uso de maneira apropriada com o propósito de auxiliar no seu desenvolvimento acadêmico. Pois esses mecanismos digitais já fazem parte da rotina dessa tríade isoladamente, com exceção de pequenas iniciativas dos próprios alunos que tentam interagir entre si de maneira informal. Visto que a maioria dos estudantes entrevistados possue tanto os aparelhos quanto os aplicativos que possibilitam esse emprego.

Vale citar como exemplo, a utilização desses mecanismos referenciados em outras Instituições, que por serem gerenciados pelos professores sob a jurisdição da faculdade, apresentam resultados positivos e expressivo diante do processo educacional.

Entretanto, é interessante que essas instituições realizem discussões com a sua comunidade, não abordando apenas os benefícios gerados com a utilização desses mecanismos, mais sim contemplando os possíveis malefícios pela prática incontrolável e viciante desses aplicativos, visto que esse estudo evidenciou uma taxa de 44,8\% dos alunos confirmam que a utilização dessas tecnologias pode trazer algum tipo de dano a sua vida pessoal. Deste modo, é recomendável mais pesquisas sobre essa ótica para que possa estar comprovando e tomando medidas adequadas para minimizar esses impactos.

Conclui-se de início que não há uma receita pronta, uma fórmula mágica para o uso consciente e disciplinado das ferramentas tecnológicas. Mas, identificar o perfil dos estudantes é um passo importante para a concretização dos objetivos. É preciso saber avaliar as preferências e os métodos de estudo. Além disso, os docentes trabalham com estudantes de diferentes personalidades, valores e culturas, podendo aprender todos os dias melhores maneiras de transmitir conhecimentos, utilizando as ferramentas tecnológicas ofertadas nos tempos atuais.

Por fim, espera que este estudo possa contribuir para o surgimento e discussão de outros estudos sobre o tema identificado, aprofundando-o de modo que este possa de fato revelar todo seu potencial como ferramenta para o bom desempenho dos estudantes.

\section{Referências}

ABREU, Cristiano Nabuco de et al. Dependência de Internet e de jogos eletrônicos: uma revisão. Rev. Bras. Psiquiatr. São Paulo, v. 30, n. 2, p. 156-167, June 2008 . 
AVILA, Rebeca Contrera. Formação das mulheres nas escolas de medicina. Rev. bras. educ. med. [online]. 2014, vol.38, n.1, pp.142-149. ISSN 0100-5502. http://dx.doi.org/10.1590/S010055022014000100019 .

BALlONE, GJ; MOURA, EC. Compulsão à Internet, Mito ou Realidade, in. PsiqWeb, Internet, disponível em <http://www.psiqweb.med.br/site/?area=NO/Ler Noticia\&idNoticia=104>. Acesso em 13 mai.2015.

BARBOSA, Ana Maria Ferrara de Carvalho et al. As novas tecnologias de comunicação: questões para a clínica psicanalítica. Cad. psicanal. [online]. 2013, v.35, n.29, p. 59-75. ISSN 1413-6295.

FREIRE, Paulo. Ação Cultural para a Liberdade e outros escritos. $6^{\mathrm{a}}$ ed. Rio de Janeiro: Paz e Terra, 1982.

IBGE, Instituto Brasileiro de Geografia e Estatística. IBGE divulga renda domiciliar per capita,. Diretoria de Pesquisas, Coordenação de Trabalho e Rendimento, Pesquisa Nacional por Amostra de Domicílios Contínua - PNAD Contínua. 2015.

IBGE, Instituto Brasileiro de Geografia e Estatística. Informações sobre Cajazeiras - Paraíba, 2010. Disponível em: http://cidades.ibge.gov.br/xtras/perfil.php?codmun =250370. Acesso em: 14 de abril de 2016.

LIMA, M. R., et al. O impacto do uso das tecnologias no aprendizado dos alunos do ensino fundamental. https://www.ufpe.br/ce/images/Graduacao_pedagogia/pdf/2007.2/o\%20impacto\%20do\%20uso\%20da s\%20tecnologias $\% 20$ no $\% 20$ aprendizado $\% 20 \mathrm{dos} \% 20$ alunos $\% 20 \mathrm{do} \% 20$ ensino $\% 20$ fundamental $\% 20 \mathrm{i} . \mathrm{p}$ df. Acessado em: 26/11/2016.

NAGUMO, Estevon. O uso do aparelho celular dos estudantes na escola. Dissertação de Mestrado. Universidade de Brasília. Faculdade de Educação. Programa de Pós-Graduação em Educação. 2014.

OLIVEIRA, Fabrícia de; Pasqualini, Kele Cristina. Os dependentes de internet no Brasil: realidade ou mito entre os universitários. Mimesis, Bauru, v. 35, n. 1, p. 95-140, 2014.

PIROCCA, Caroline. Dependência de internet, definição e tratamentos: revisão sistemática da literatura. Monografia apresenta ao Instituto de Psicologia da Universidade Federal do Rio Grande do Sul. 2012

SCHEFFER, Mário César and CASSENOTE, Alex Jones Flores. A feminização da medicina no Brasil. Rev. Bioét. [online]. 2013, v.21, n.2, pp.268-277. ISSN 19838042. http://dx.doi.org/10.1590/S1983-80422013000200010.

SETZER, VALDEMAR W. EFEITOS NEGATIVOS DOS MEIOS ELETRÔNICOS EM CRIANÇAS, ADOLESCENTES E ADULTOS. Depto. de Ciência da Computação, Instituto de Matemática e Estatística da USP. 2014. Disponível em: https://www.ime.usp.br/ vwsetzer/efeitosnegativos-meios.html.

SMITH, Craig. By the Numbers: 40. Amazing WhatsApp Statistics. Disponível em: <http://expandedramblings.com/index.php/whatsapp-statistics>. Acesso em: 20 março de 2016.

SOUZA, Maria C. C. Tecnologias avançadas e mudanças de paradigmas. Em:http://www.telecomuff.com Disponível em, 
http://www.telecomuff.com/uploads/6/9/4/8/6948141/uff_-

_tecnologias_avanadas_e_mudanas_de_paradigmas_-_2014_-_sp.pdf , 20/11/2016

TEIXEIRA, Carina Rabelo Dias; KER, Mariana Gama; MAGALHÃES, Evaristo Nunes de. AspectosPsicopatologicos-da-Dependência-da-Internet. Psicologado Artigos. Disponível em: https://psicologado.com/psicopatologia/transtornos-psiquicos/aspectos-psicopatologicos-dadependencia-da-internet. Acesso em: 20/11/2016.

TEIXEIRA, Vany Regina et al. Evolução histórica do processo ensino - aprendizagem. Secretaria de Educação do Estado de Mato Grosso. [online], 2015.

\section{Como citar este artigo (Formato ABNT):}

BARBOSA, Sylvio E. da S.; DO CARMO, Larissa A.; MEDEIROS, Renata L.S.F.M de; CABRAL, Symara A. A. de O. ; LOPES, Raimundo Edilberto M.; ALVES, Maria Nizete T. Impacto na Rotina Acadêmica pela Utilização de Novas Tecnologias por Estudantes de Medicina do Semiárido Paraibano. Id on Line Revista Multidisciplinar e de Psicologia, 2018, vol.12, n.39, p.695-712. ISSN: 1981-1179.

Recebido: 19.01 .2018

Aceito: 26.01 .2018 